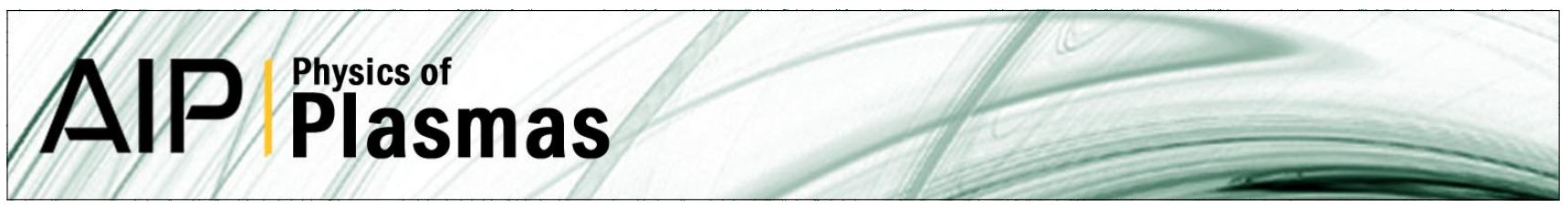

\title{
Gyrokinetic linear theory of the entropy mode in a Z pinch
}

Paolo Ricci, B. N. Rogers, W. Dorland, and M. Barnes

Citation: Phys. Plasmas 13, 062102 (2006); doi: 10.1063/1.2205830

View online: http://dx.doi.org/10.1063/1.2205830

View Table of Contents: http://pop.aip.org/resource/1/PHPAEN/v13/i6

Published by the American Institute of Physics.

\section{Related Articles}

Implications for the electron distribution from the stationary hydrodynamic model of a one-dimensional plasma expansion into vacuum

Phys. Plasmas 19, 102101 (2012)

Magneto-modulational instability in Kappa distributed plasmas with self-generated magnetic fields

Phys. Plasmas 19, 092114 (2012)

Langmuir probe diagnostics of an atmospheric pressure, vortex-stabilized nitrogen plasma jet

J. Appl. Phys. 112, 063302 (2012)

Kinetic theory of magnetized dusty plasmas with dust particles charged by collisional processes and by photoionization

Phys. Plasmas 19, 093702 (2012)

Kinetic model of electric potentials in localized collisionless plasma structures under steady quasi-gyrotropic conditions

Phys. Plasmas 19, 082904 (2012)

\section{Additional information on Phys. Plasmas}

Journal Homepage: http://pop.aip.org/

Journal Information: http://pop.aip.org/about/about_the_journal

Top downloads: http://pop.aip.org/features/most_downloaded

Information for Authors: http://pop.aip.org/authors

\section{ADVERTISEMENT}

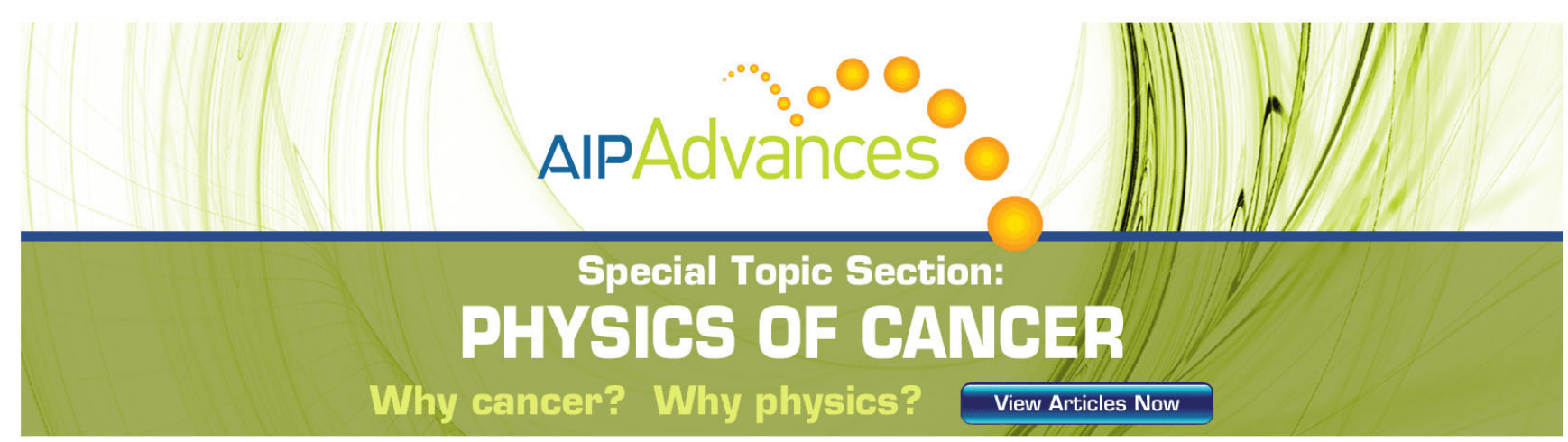




\title{
Gyrokinetic linear theory of the entropy mode in a Z pinch
}

\author{
Paolo Riccia) and B. N. Rogers ${ }^{\text {b) }}$ \\ Department of Physics and Astronomy, Dartmouth College, Hanover, New Hampshire 03755 \\ W. Dorland ${ }^{\mathrm{C})}$ and M. Barnes ${ }^{\mathrm{d})}$ \\ Department of Physics, University of Maryland, College Park, Maryland 20742
}

(Received 20 March 2006; accepted 26 April 2006; published online 12 June 2006)

\begin{abstract}
The linear gyrokinetic theory of the entropy mode is presented in a Z-pinch configuration in the regime of plasma $\beta \ll 1$, focusing primarily on the parameter regime in which the ideal interchange mode is stable. The entropy mode is a small-scale, nonmagnetohydrodynamic mode that typically has peak growth rates at $k \rho_{s} \sim 1\left[\rho_{s}^{2}=\left(T_{0 e}+T_{0 i}\right) /\left(m_{i} \Omega_{c i}^{2}\right)\right]$, with magnitudes comparable to those of ideal modes. The properties of this mode are studied as a function of the density and temperature gradients, plasma collisionality, and electron to ion temperature ratio. (C) 2006 American Institute of Physics. [DOI: 10.1063/1.2205830]
\end{abstract}

\section{INTRODUCTION}

This paper considers the kinetic theory of small-scale linear instabilities in a Z-pinch configuration, in which a cylindrically symmetric plasma is confined by a poloidal magnetic field $B_{\theta}(r) .{ }^{1}$ We focus here on the regime of plasma $\beta \ll 1$, in which the dominant instabilities in the Z-pinch system, driven by a combination of the pressure gradient and magnetic curvature, take on an electrostatic character and have $k_{\|}=0 .{ }^{2}$ Past studies of this system, ${ }^{2-7}$ mainly carried out under the assumption of long wavelength in comparison to the ion gyroradius, $k \rho_{i} \ll 1\left(\rho_{i}\right.$ being the ion gyroradius and $k=k_{\perp}$ ), have identified the possible presence of two distinct linear modes. At sufficiently steep gradients, the fastest growing mode is the well-known ideal magnetohydrodynamic (MHD) interchange instability with $\gamma \sim c_{s} / \sqrt{R L_{p}}\left[c_{s}\right.$ $=\sqrt{\left(T_{0 e}+T_{0 i}\right) / m_{i}}$ being the sound speed, $R$ the curvature radius, and $L_{p}=-p_{0} / p_{0}^{\prime}$, with $p_{0}^{\prime}=d p_{0} / d r$, an equilibrium pressure-gradient scale length that is assumed to be positive, since we focus our attention on $\mathrm{Z}$ pinches where the pressure decreases with the radius]. ${ }^{8,9}$ At weaker gradients the ideal mode can be stabilized, however, leaving behind a shorter wavelength non-MHD mode known as the entropy mode. This mode, introduced in the 1960s by the works of several authors ${ }^{10-13}$ and also referred to in the literature as the drifttemperature-gradient mode, ${ }^{5,6}$ perturbs both the plasma density and temperature but not the total plasma pressure, and hence the specific entropy of the plasma is changed. The entropy mode has been studied more recently in the linear regime using both fluid ${ }^{3}$ and (under the assumption of $k \rho_{i}$ $\ll 1)$ gyrokinetic models. ${ }^{2,5-7}$ These gyrokinetic analytical calculations were performed in the regime with equal electron and ion temperatures, $T_{0 i}=T_{0 e}$ in Refs. 2, 5, and 6 and generic temperature ratio in Ref. 7, and it was found that the growth rate of the entropy mode increases linearly with $k$. As

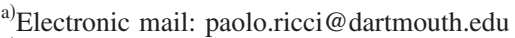

${ }^{b)}$ Electronic mail: barrett.rogers@dartmouth.edu

${ }^{c)}$ Electronic mail: bdorland@umd.edu

d)Electronic mail: mabarnes@umd.edu
}

a result, the most unstable regime of the mode could not be addressed.

The primary goal of this paper is to explore the linear theory of the entropy mode in ideally stable parameter regimes for arbitrary $k \rho_{i}$, various levels of density and temperature gradients, plasma collisionality, and ion to electron temperature ratios. The saturation of the linear modes and the nonlinear dynamics of the $\mathrm{Z}$ pinch will be the subject of a future paper. Here the study of the linear modes is accomplished through the numerical solution of the gyrokinetic equations developed in Refs. 14 and 15. We use the code GS2 to solve the gyrokinetic system and evaluate the growth rate of the linear modes ${ }^{16,17}$ and, as a benchmark for GS2, we also perform the direct solution of the gyrokinetic dispersion relation in the simplest cases. Although the gyrokinetic equations have been studied analytically for $k \rho_{i} \ll 1,2,5-7$ the present study (to our knowledge) is the first to explore the regime of $k \rho_{i} \sim 1$ where the modes are most unstable.

Our analysis presupposes that $\rho_{i}$, as well as the (perpendicular) scale lengths of the modes of interest (e.g., $1 / k$ ), are both small compared to equilibrium scale lengths such as $L_{p}$ and $R$. Given this, our kinetic calculations show that the entropy mode is unstable at a gradient about two times lower than the marginal stability gradient predicted by the fluid theory. The growth rate spectrum of the entropy mode as a function of $k$ typically peaks at $k \rho_{s} \sim 1\left(\rho_{s}=c_{s} / \Omega_{c i}\right.$, being $\Omega_{c i}$ the ion cyclotron frequency), where it reaches a maximum value that is typically comparable to the growth rate of the ideal interchange mode. Such large growth rates suggest that the entropy mode, in its nonlinear phase, may potentially drive significant particle and heat transport in ideally stable $\mathrm{Z}$ pinches. The instability can extend well into the regime of $k \rho_{i}>1$, making a kinetic treatment valid to all orders in $k \rho_{i}$ necessary for an accurate description for $T_{0 i} \gtrless T_{0 e}$.

This paper is organized as follows. In the next section we discuss the so-called local approximation on which our calculations are based, and review the MHD equilibrium condition in a $\mathrm{Z}$ pinch. In Sec. III, we then summarize some important results for the ideal interchange and the entropy modes that have been obtained in the literature in the limit of 
$k \rho_{i} \ll 1$. For convenience, these results are also derived in Appendix A from the gyrofluid model. In Sec. IV we then turn to the numerical study of the gyrokinetic model and the case of arbitrary $k \rho_{i}$. The conclusions are given in Sec. V.

\section{THE LOCAL APPROXIMATION}

Since the modes of interest have scale lengths that are much smaller than those of the equilibrium quantities, the analysis described in this paper, like most previous studies of the entropy mode, are carried out within the framework of the so-called local approximation. ${ }^{18}$ In this approximation, one first separates the dependent variables into equilibrium parts (if present) and perturbations. Given the assumed disparity in the scale-lengths between the two parts, one may then Taylor expand the equilibrium quantities about some radius of interest $r=R$ and retain only the dominant, leadingorder terms. As a result, equilibrium quantities appearing in the final equations for the perturbations, such as the curvature radius $R$, the magnetic field $\mathbf{B}=B_{\theta} \mathbf{e}_{\theta}$, the density $n_{0}$, the ion and electron temperatures, $T_{0 i}$ and $T_{0 e}$, the equilibrium scale lengths $L_{n}=-n_{0} / n_{0}^{\prime}, L_{p}=-p_{0} / p_{0}^{\prime}, L_{T_{\alpha}}=-T_{0 \alpha} / T_{0 \alpha}^{\prime}$, etc., are approximated as constants within the narrow domain under consideration. These quantities are assumed to be related to the condition for MHD equilibrium, which in the Z-pinch geometry may be written as ${ }^{1}$

$$
\frac{\beta}{L_{p}}=\frac{2}{r B_{\theta}} \frac{d}{d r}\left(r B_{\theta}\right) .
$$

In the limit of $\beta \ll 1$, the main interest in this paper, it is seen that to leading order in $\beta$ the magnetic field strength $B_{\theta}$ $\propto 1 / r$ as in a vacuum, and the quantities $R$ and $L_{p}$ may be regarded as essentially independent, constant parameters. Unless otherwise noted, for simplicity we will assume here that $L_{n_{i}}=L_{n_{e}}=L_{n}$ and $L_{T_{i}}=L_{T_{e}}=L_{T}$.

\section{REVIEW OF PAST RESULTS AT $k \rho_{i} \ll 1$}

As noted in the Introduction, our study addresses the linear theory of the entropy mode in parameter regimes in which the ideal interchange mode is stable. In this section we therefore review the dispersion relation for the ideal interchange mode in the collisional and collisionless limits. We then turn to a discussion of the entropy mode in the limit $k \rho_{i} \ll 1$ in which some analytical progress can be made. The full gyrokinetic theory of the entropy mode for arbitrary $k \rho_{i}$ is discussed in the following section.

The numerical solution of the electromagnetic gyrokinetic dispersion relation carried out in three spatial dimensions show that for $\beta \ll 1$ the fastest growing modes are electrostatic and have $k_{\|}=0$ and $k_{r}=0$. Thus, all the analytical calculations and the numerical studies presented in the present paper are performed in the electrostatic approximation and neglect the dependence of the modes on the azimuthal and radial coordinates.

\section{A. Interchange mode}

In the limit of $k \rho_{i} \ll 1, \beta \ll 1$, and strong collisionality, the dispersion relation for the standard ideal MHD interchange mode can be written as (see, e.g., Ref. 5)

$$
\begin{aligned}
& \left(k \rho_{s e}\right)^{2} \omega^{2}-2 \omega_{d e}\left[\frac{10}{3}\left(\omega_{d e}-\omega_{d i}\right)-(1+\eta)\left(\omega_{*_{e}}-\omega_{*_{i}}\right)\right] \\
& \quad=0
\end{aligned}
$$

where $\eta=L_{n} / L_{T}, \rho_{\text {se }}$ the ion Larmor radius based on the electron temperature, and the general expression for $\omega_{* \alpha}$ and $\omega_{d \alpha}$ take on a simple form in a $\mathrm{Z}$ pinch:

$$
\begin{aligned}
& \omega_{* \alpha}=-\frac{c T_{0 \alpha}}{q_{\alpha} B n_{0}} \nabla n_{0} \cdot \mathbf{b} \times \mathbf{k}=k \rho_{\alpha} \frac{v_{\mathrm{th}, \alpha}}{L_{n}}, \\
& \omega_{d \alpha}=\frac{v_{\mathrm{th}, \alpha}^{2}}{\Omega_{c \alpha} B^{2}} \mathbf{B} \times \nabla B \cdot \mathbf{k}=k \rho_{\alpha} \frac{v_{\mathrm{th}, \alpha}}{R},
\end{aligned}
$$

b being the unit vector of the magnetic field $\mathbf{B}$, and $q_{\alpha}, v_{\mathrm{th}, \alpha}$, $\Omega_{c \alpha}=q_{\alpha} B_{\theta} /\left(m_{\alpha} c\right), m_{\alpha}$, and $\rho_{\alpha}=v_{\text {th }, \alpha} / \Omega_{c \alpha}$ being the charge, thermal velocity, cyclotron frequency, mass, and gyroradius for the species $\alpha$, respectively (with the above definitions $\left.\rho_{e}<0\right)$. It is seen that the term in Eq. (2) proportional to $\omega_{d e}\left(\omega_{*_{e}}-\omega_{*_{i}}\right)$ drives the growth of the mode while the term proportional to $\omega_{d e}\left(\omega_{d e}-\omega_{d i}\right)$, arising from the contribution of the plasma compressibility, is always stabilizing. In the limit that the former dominates, one obtains $\gamma$ $\sim \sqrt{\omega_{d e}\left(\omega_{* e}-\omega_{*_{i}}\right)(1+\eta)} /\left(k \rho_{s e}\right) \sim c_{s} / \sqrt{R L_{p}}$, as noted in the Introduction. The stability region associated with Eq. (2) as a function of $\left(\eta, L_{n} / R\right)$ is shown in Fig. 1. In the case of $\eta$ $=0$ and $\tau=1\left(\tau=T_{0 i} / T_{0 e}\right)$, the collisional interchange mode is unstable for $0<L_{n} / R<3 / 10$. For convenience, Eq. (2) is derived in Appendix A from the gyrofluid model. ${ }^{18-20}$

The dispersion relation for the interchange mode in a collisionless plasma can be derived from the Chew, Goldberger, and Low (CGL) model, ${ }^{21}$ assuming zero heat flow perpendicular to the magnetic field. Aside from numerical factors, the resulting dispersion relation is similar to that of the collisional case and can be written as: ${ }^{22}$

$$
\left(k \rho_{s e}\right)^{2} \omega^{2}-\omega_{d e}\left[7\left(\omega_{d e}-\omega_{d i}\right)-2(\eta+1)\left(\omega_{*_{e}}-\omega_{*_{i}}\right)\right]=0 .
$$

The stability region of the collisionless interchange mode in the $\left(\eta, L_{n} / R\right)$ plane is shown in Fig. 1. For $\eta=0$ and $\tau=1$, the collisionless interchange mode is unstable for $0<L_{n} / R$ $<2 / 7(\simeq 0.29)$. Equation (5) is also derived in Appendix A from the gyrofluid model.

\section{B. Entropy mode}

In the limit of $k \rho_{i} \ll 1$ and $\tau=1$, the dispersion relation for the entropy mode in collisional plasmas has been obtained in Refs. 5-7; for convenience, it is also derived in Appendix A from the gyrofluid model for arbitrary temperature ratio: 
a)

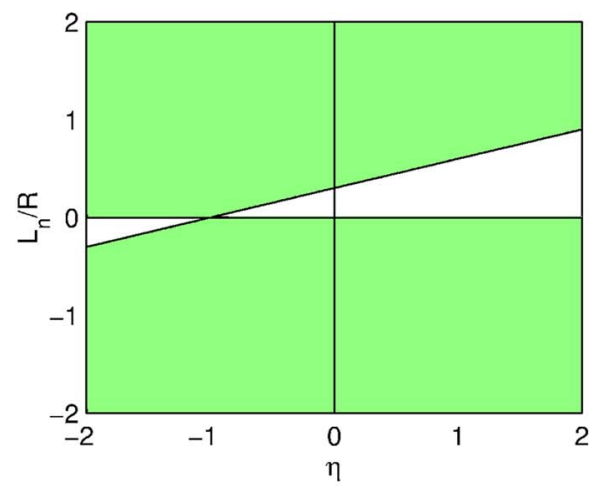

c)

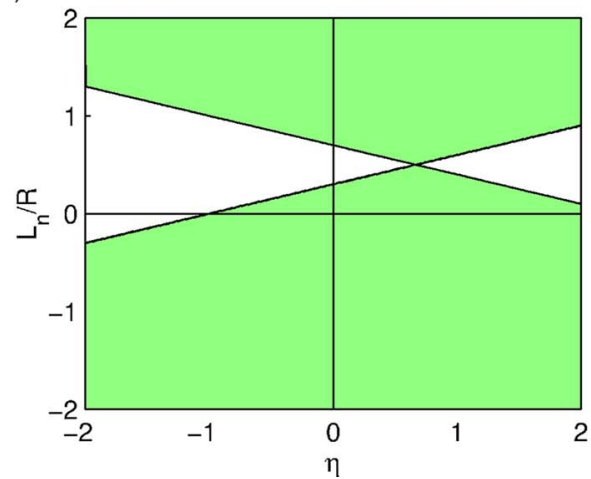

b)

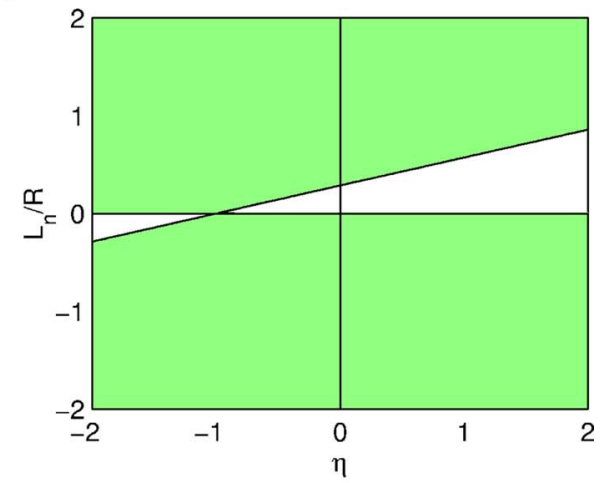

d)

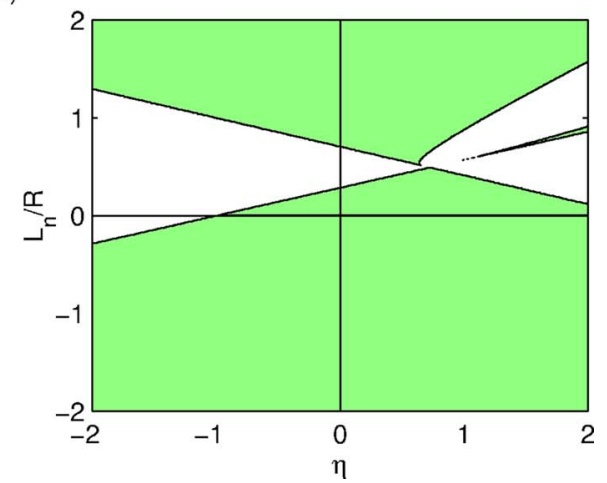

FIG. 1. (Color online) Stability region for the interchange mode in the collisional, Eq. (2) (a), and collisionless regime, Eq. (5) (b), and for the entropy mode in the collisional, Eq. (6) (c), and collisionless regime, Eq. (8) (d), in the $\left(L_{n} / R, \eta\right)$ plane. The white regions represent the region where the modes are unstable.

$$
\begin{aligned}
& {\left[9(1+\eta)\left(\omega_{*_{e}}-\omega_{*_{i}}\right)+30\left(\omega_{d i}-\omega_{d e}\right)\right] \omega^{2}-30\left[2 \left(\omega_{d i}^{2}\right.\right.} \\
& \left.\left.-\omega_{d e}^{2}\right)-\left(\omega_{d i} \omega_{*_{i}}-\omega_{d e} \omega_{*_{e}}\right)\right] \omega+20 \omega_{d e}\left(\omega_{d i}-\omega_{d e}\right) \\
& \quad \times\left[10 \omega_{d i}+\omega_{*_{i}}(3 \eta-7)\right]=0
\end{aligned}
$$

which leads to the scaling

$$
\gamma \sim \sqrt{\frac{\omega_{d e}\left(\omega_{d i}-\omega_{d e}\right)\left[10 \omega_{d i}+\omega_{* i}(3 \eta-7)\right]}{9(1+\eta)\left(\omega_{*}-\omega_{*_{i}}\right)+30\left(\omega_{d i}-\omega_{d e}\right)}} \sim \frac{k \rho_{s e} v_{\mathrm{th}, i}}{R}
$$

for $\tau \sim 1$, thus showing the linear dependence of the growth rate on $k$ noted in the Introduction. In the limit of $\eta=0$ and $\tau=1$, for example, instability is obtained for $3 / 10<L_{n} / R$ $<7 / 10$. In the collisionless limit (and $\tau=1$ for simplicity), on the other hand, the gyrofluid model yields

$$
\begin{aligned}
& \omega_{d}\left[7 \omega_{d}-2(\eta+1) \omega_{*}\right] \omega^{4}-\left[221 \omega_{d}^{4}-2(19 \eta+44) \omega_{d}^{3} \omega_{*}\right] \omega^{2} \\
& +2\left[578 \omega_{d}^{6}+34(5 \eta-12) \omega_{d}^{5} \omega_{*}\right]=0,
\end{aligned}
$$

where $\omega_{*}=\omega_{*_{i}}=-\omega_{*_{e}}$ and $\omega_{d}=\omega_{d i}=-\omega_{d e}$. For $\eta=0$, the collisionless entropy mode becomes unstable in the presence of lower gradients than the ideal interchange mode, namely $2 / 7<L_{n} / R<204 / 289(\simeq 0.71)$ for $\tau=1$. The stability region of both the collisional and the collisionless entropy mode, Eqs. (6) and (8), are shown in Fig. 1 for $\tau=1$.

In Fig. 2, the growth rates computed with the dispersion relations of the ideal interchange mode [Eqs. (2) and (5)] and of the entropy mode [Eqs. (6) and (8)] are compared with the growth rates obtained from the dispersion relation of the gy- rofluid model, Eq. (A15), that couples the ideal interchange and the entropy modes, and from the gyrokinetic model, described later. For clarity, the dispersion relations corresponding to the different models are summarized in Table I. In Fig. 2 , we consider small values of $k \rho_{s},\left(k \rho_{s}\right)^{2}=0.002$, and $\left(k \rho_{s}\right)^{2}=0.2$, and the growth rates are plotted as a function of $L_{n} / R \quad(\eta=0$ and $\tau=1)$. In the case of $\left(k \rho_{s}\right)^{2}=0.002$, the growth rates both from the gyrokinetic and the gyrofluid models agree well with the analytical estimate from the ideal dispersion relation at high density gradient, in the ideal interchange regime. We note that, at the weakest gradients (e.g., in the range of $L_{n} / R$ from about 0.7 to $\pi / 2$ ), the gyrokinetic model exhibits instability while the gyrofluid model does not. For $\eta=0$, the value of the gyrokinetic stability threshold, $L_{n} / R=\pi / 2$, can be analytically evaluated and the details of the derivation are presented in Appendix B.

\section{GYROKINETIC LINEAR THEORY}

\section{A. Gyrokinetic model}

The gyrokinetic equations ${ }^{14,15}$ described in detail below are based on the ordering $\rho_{i} \ll L$ (where $L$ is a generic equilibrium scale), $k L \gg 1$, and $\omega \ll \Omega_{c i}$. Given that the dominant modes in our system typically have $k \sim 1 / \rho_{i}$ for $\tau \sim 1$, the condition $k L \gg 1$ is well satisfied provided that $\rho_{i} \ll L$. Likewise, since the maximum frequencies in our system are typically on the order of the ion or electron diamagnetic frequen- 

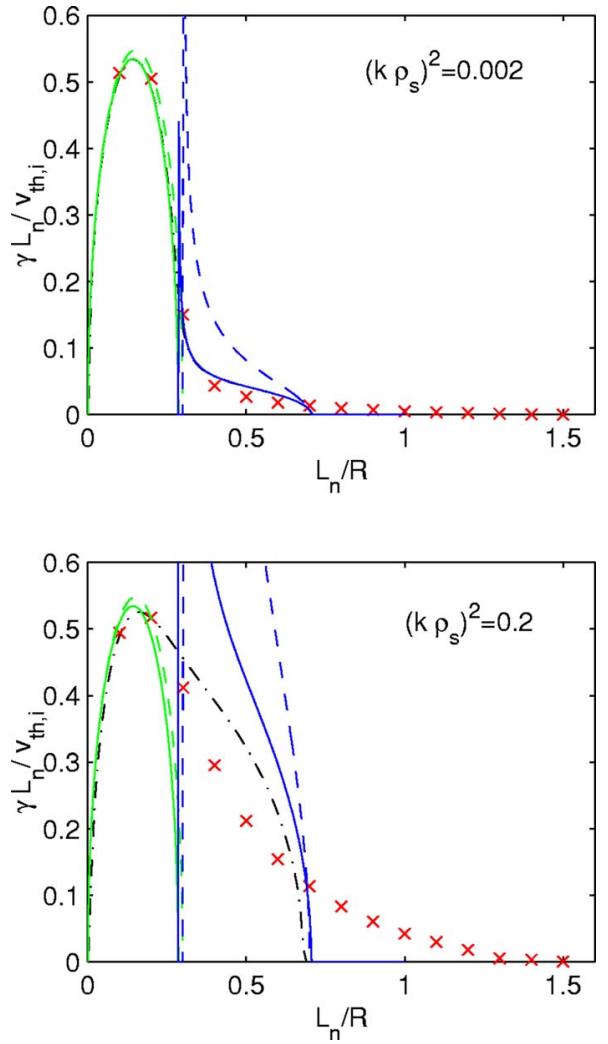

FIG. 2. (Color online) Growth rate of the interchange and the entropy mode as a function of $L_{n} / R$ for two different values of $k \rho_{s}(\eta=0, \tau=1$, and $\nu=0)$. The gyrokinetic growth rate (red " $X$ " marks) is compared to the growth rate from the gyrofluid model (black dashed-dotted line), Eq. (A15), from the dispersion relation of the ideal collisional (green dashed line) and collisionless interchange mode (green solid line), Eqs. (2) and (5), and of the collisional (blue dashed line) and collisionless entropy mode (blue solid line), and Eqs. (6) and (8). In GS2 calculation it is assumed that $m_{i} / m_{e}=1836$.

cies $\left(\omega \sim \omega_{*}\right)$, the condition $\Omega_{c i} \gg \omega$ for $\tau \sim 1$ reduces to $L / \rho_{i} \gg k \rho_{i}$ and is thus also satisfied provided that $L / \rho_{i}$ is sufficiently large.

Assuming all the perturbed quantities are proportional to $\exp (-i \omega t+i k z)$, the gyrokinetic equations for both ions and electrons can be written in the following form:

$$
\begin{aligned}
\left(\omega-\Omega_{d \alpha}\right) h_{\alpha}= & -\left(\omega-\Omega_{*_{\alpha}}\right) q_{\alpha} \phi \frac{\partial F_{0 \alpha}}{\partial \epsilon} J_{0}\left(\frac{k v_{\perp}}{\Omega_{c \alpha}}\right) \\
& +\frac{i}{2 \pi} \int_{-\pi}^{\pi} \exp (-i L) C\left(f_{\alpha}\right) d \xi
\end{aligned}
$$

where $L=(\mathbf{v} \times \mathbf{b} \cdot \mathbf{k}) / \Omega_{c \alpha}, \xi$ is the gyroangle, $\phi$ is the electrostatic potential, and the distribution function $h_{\alpha}=h_{\alpha}(\epsilon, \mu)$, with $\epsilon=m_{\alpha} v^{2} / 2$ and $\mu=m_{\alpha} v_{\perp}^{2} / 2 B_{\theta}$, is related to the total perturbed distribution function $f_{\alpha}=f_{\alpha}(\mathbf{v})$ through

$$
f_{\alpha}=q_{\alpha} \phi \frac{\partial F_{0 \alpha}}{\partial \epsilon}+\exp (i L) h_{\alpha}
$$

The frequencies $\Omega_{d \alpha}$ and $\Omega_{* \alpha}$ are related to the frequencies $\omega_{d \alpha}$ and $\omega_{* \alpha}$ by

$$
\begin{aligned}
\Omega_{* \alpha}= & \frac{\mathbf{b} \times \mathbf{k} \cdot \nabla F_{0 \alpha}}{m_{\alpha} \Omega_{c \alpha} \partial F_{0 \alpha} / \partial \epsilon}=\omega_{* \alpha}\left(\frac{L_{n} F_{0 \alpha} n_{0}^{\prime} / n_{0}}{T_{0 \alpha} \partial F_{0 \alpha} / \partial \epsilon}\right. \\
& \left.+\frac{L_{n} n_{0}\left(F_{0 \alpha} / n_{0}\right)^{\prime}}{T_{0 \alpha} \partial F_{0 \alpha} / \partial \epsilon}\right), \\
\Omega_{d \alpha}= & \mathbf{k} \cdot \mathbf{b} \times \frac{m_{\alpha} v_{\|}^{2} \mathbf{b} \cdot \nabla \mathbf{b}+\mu \nabla B}{m_{\alpha} \Omega_{c \alpha}}=\omega_{d \alpha}\left(\frac{v_{\|}^{2}+v_{\perp}^{2} / 2}{v_{\mathrm{th}, \alpha}^{2}}\right) .
\end{aligned}
$$

The collision operator used in the present paper, $C\left(f_{\alpha}\right)$, takes into account electron-electron, electron-ion, and ionion collisions and is expressed as ${ }^{16}$

$$
C\left(f_{\alpha}\right)=\hat{\nu}(v) \frac{\partial}{\partial \xi}\left(1-\xi^{2}\right) \frac{\partial f_{\alpha}}{\partial \xi},
$$

where $\xi=v_{\|} / v$ and $\hat{\nu}(v)$ is defined in Ref. 16 . We assume for simplicity that both the ion and electron equilibrium distribution functions are isotropic Maxwellians,

$$
F_{0 \alpha}=n_{0}\left(\frac{m_{\alpha}}{2 \pi T_{0 \alpha}}\right)^{3 / 2} \exp \left(-\frac{\epsilon}{T_{0 \alpha}}\right) .
$$

The ion and electron gyrokinetic distribution functions are coupled by the quasineutrality equation

$$
\sum_{\alpha} q_{\alpha} \int f_{\alpha} d \mathbf{v}=0
$$

We use GS2 (see Refs. 16 and 17) to solve the gyrokinetic system, Eqs. (9) and (15), and evaluate the growth rate of the linear modes. The code evolves the five-dimensional perturbed distribution functions $f_{\alpha}(\mathbf{r}, \epsilon, \mu)$ on a fixed grid. The linear modes are evaluated with an initial value approach: small initial noise is evolved until the eigenfunction with the fastest growth rate is formed. As a benchmark for GS2, we also solve the linear gyrokinetic dispersion relation with an interior-reflective Newton iterative method, ${ }^{23}$ evaluating the integrals present in the gyrokinetic dispersion relation with an adaptive Simpson quadrature method.

\section{B. Numerical results}

We have explored the growth rate of the entropy mode as a function of $k$ for different values of $L_{n} / R, \eta$, collision frequency $\nu\left[\nu=\pi n_{0} e^{4} \ln \Lambda /\left(m_{i}^{1 / 2} T_{0 i}^{3 / 2}\right)\right.$, where $\ln \Lambda$ is the Coulomb logarithm], and $\tau\left(\tau=T_{0 i} / T_{0 e}\right)$. In Fig. 3, we show

TABLE I. Summary of the different models used in the study of the linear theory of the $Z$ pinch.

\begin{tabular}{lcccc}
\hline \hline & Interchange & Entropy $\left(k \rho_{i} \ll 1\right)$ & Interchange/Entropy $\left(k \rho_{i} \ll 1\right)$ & Entropy \\
\hline Collisional & MHD, Eq. (2) & Gyrofluid, Eq. (6) & Gyrofluid, Eq. (A9) & Gyrokinetic, Eqs. (9) and (15) \\
Collisionless & CGL, Eq. (5) & Gyrofluid, Eq. (8) & Gyrofluid, Eq. (A15) & Gyrokinetic, Eqs. (9) and (15), $C\left(h_{\alpha}\right)=0$ \\
\hline \hline
\end{tabular}




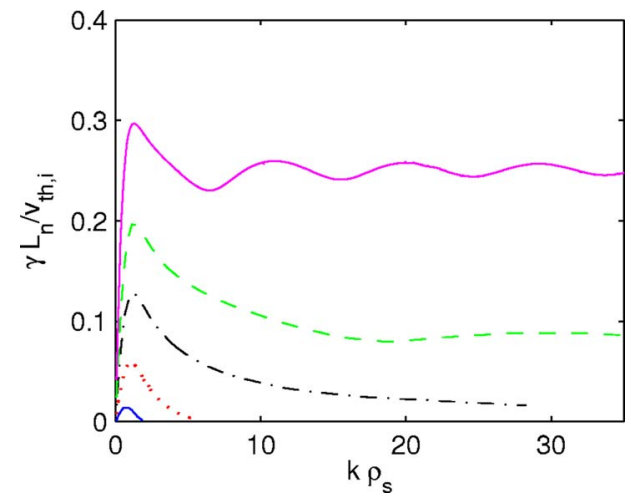

FIG. 3. (Color online) Growth rate of the entropy mode as a function of $k \rho_{s}$ for different values of $L_{n} / R: L_{n} / R=1.25$ (blue solid line), $L_{n} / R=1$ (red dotted line), $L_{n} / R=0.8$ (black dashed-dotted line), $L_{n} / R=0.67$ (green dashed line), and $L_{n} / R=0.5$ (magenta solid line). It is assumed $\eta=0, \tau=1$, and $m_{e}=0$.

the growth rate of the entropy mode as a function of $k$, for different values of $L_{n} / R(\tau=1, \nu=0)$. The growth rates are proportional to $k$ for $k \rho_{s}<1$, as predicted by the analytical dispersion relation of the entropy mode, Eq. (8). The peak growth rate is found to be at $k \rho_{s} \sim 1$ and increases considerably with $L_{n} / R$ (it passes from $\gamma \simeq 0.013 v_{\text {th }, i} / L_{n}$ in the case of $L_{n} / R=1.25$ to $\gamma \simeq 0.3 v_{\text {th }, i} / L_{n}$ at $\left.L_{n} / R=0.5\right)$. The cutoff at high $k$ increases considerably with the gradient. For $L_{n} / R$ $=0.8$, the cutoff is over $k \rho_{s} \sim 30$, while for $L_{n} / R=0.5$ the growth rate does not seem to present a cutoff at all up to the value of $k \rho_{i} \sim 70$ that we have examined. As an aside, we note that the numerical solution of the gyrokinetic equations at $k \rho_{i} \simeq 70$, where $k \rho_{e} \simeq 1.6$ (for $m_{i} / m_{e}=1836$ ), shows that electron finite Larmor radius (FLR) effects reduce the growth rate of the entropy mode, although they are not able to stabilize it completely.

As a benchmark for GS2, we describe some convergence studies with respect to the GS2 energy grid. In Fig. 4 we plot the growth rate of the entropy mode for $L_{n} / R=0.95$ ( $\eta=0$, $\nu=0$, and $\tau=1$ ) for different numbers of grid points in the velocity space, and we compare with the direct numerical

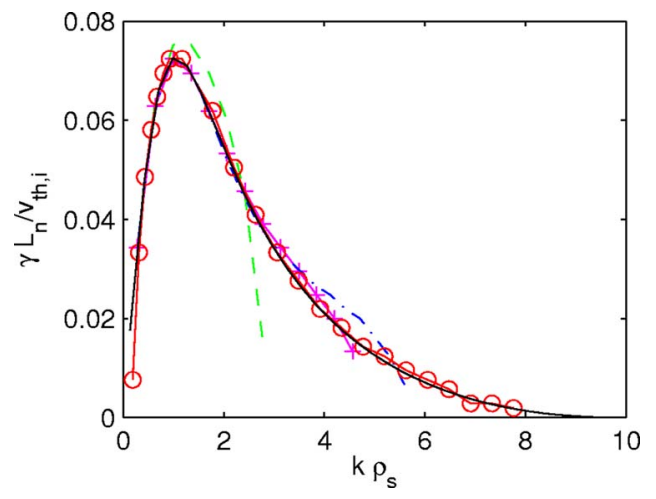

FIG. 4. (Color online) The growth rate of the entropy mode for the case of $L_{n} / R=0.95$ and $\eta=0$ is plotted as computed from GS2 with different numbers of grid points in velocity space: 60 points (green dashed line), 360 points (magenta line with + marks), 420 points (blue dashed-dotted line), and 16384 points (red line with " $\bigcirc$ " marks). The black solid lines shows the direct numerical solution. The convergence test is performed for $m_{e}=0, \tau$ $=1, \nu=0$, and $\eta=0$. solution of the gyrokinetic dispersion relation. The agreement between the growth rate is good at low $k$ for all the velocity grids, but the comparison reveals that only refined velocity grids are able to represent correctly the growth rate at $k \rho_{s} \geqslant 4$. Convergence tests also show that the number of grid points in the velocity space necessary to evaluate the correct growth rate of the entropy mode is bigger for density gradients close to the stability threshold of $L_{n} / R=\pi / 2$ (for $\eta=0$ ). This is due to the fact that the dependence of the distribution functions on $v_{\|}$and $v_{\perp}$ requires a refined energy grid to be well represented at high $k$ and at weak gradient. This is shown in Fig. 5, where the ion and electron distribution functions are plotted for $k \rho_{s}=38\left(L_{n} / R=0.5\right)$, revealing a high frequency oscillation of the ion distribution function in both the $v_{\perp}$ and $v_{\|}$directions. As an aside, we note that a new algorithm to evaluate the energy grid points for Gaussian integration has been implemented in GS2 (Ref. 24) in order to perform calculations with a refined velocity grid. ${ }^{25}$

The importance of the temperature gradient for the entropy mode is analyzed in Fig. 6 where we plot the growth rate of the mode as a function of $k$ for different values of $L_{n} / R$ and $\eta(\nu=0$ and $\tau=1)$. As it is possible to argue also from the stability region of the entropy mode (Fig. 1), positive values of $\eta$ are stabilizing for the entropy mode, while negative values of $\eta$ are destabilizing. For low values of $L_{n} / R$ (high density gradient) both the peak value and the growth rate at high $k$ are affected by $\eta$; at high values of $L_{n} / R$ (weak density gradient) only the growth rate at high $k$ is affected.

We also perform a study of the growth rate of the entropy mode as a function of the plasma collisionality, using the collision operator of Eq. (13) that includes electronelectron, electron-ion, and ion-ion collisions. In Fig. 7, we plot the growth rate of the entropy mode as a function of $k$ for different plasma collisionality $\nu$ and $L_{n} / R$, in the case of $\eta=0, \tau=1$. Collisions decrease the growth rate of the entropy mode at high values of $k$, since collisions tend to suppress the formation of anisotropies in the distribution function, as the one shown in Fig. 4, that are typical of the entropy mode at high $k$. Moreover, in the case of low gradient, where kinetic effects lead the instability (see Fig. 2), collisions are also able to decrease the peak value of the growth rate.

The effect of the temperature ratio, $\tau$, on the growth rate of the entropy mode is shown in Fig. $8(\eta=0, \nu=0)$. The numerical results show two different dependences of the growth rate on the temperature ratio, depending on the density gradient. At high gradient, close to the ideal interchange stability, the growth rate for $k \rho_{s}<1$ scales with $k \rho_{s e}$, consistent with Eq. (7). The peak growth rate is localized at $k \rho_{s}$ $\sim 1$ (this finding has been verified with $\tau$ that ranges from $\tau=0.1$ to $\tau=20$ ). Also, the peak growth rate decreases with $\tau$. At lower gradients, closer to the entropy mode marginal stability boundary, for $\tau>1$, the peak growth rate is shifted to shorter wavelength and the peak tends to broaden, so that the entropy mode becomes unstable up to very high values of $k \rho_{i}$. The growth rate is smaller than the $\tau=1$ case, for both $\tau>1$ and $\tau<1$. We remark that also the growth rate near marginal stability, evaluated with the assumption $k \rho_{i} \ll 1$ and 
a)

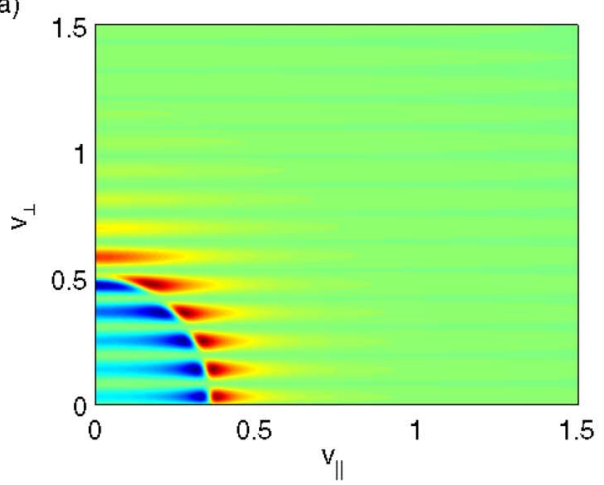

c)

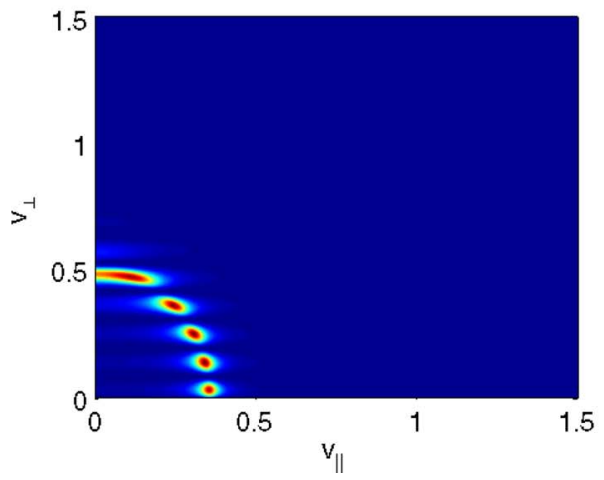

b)

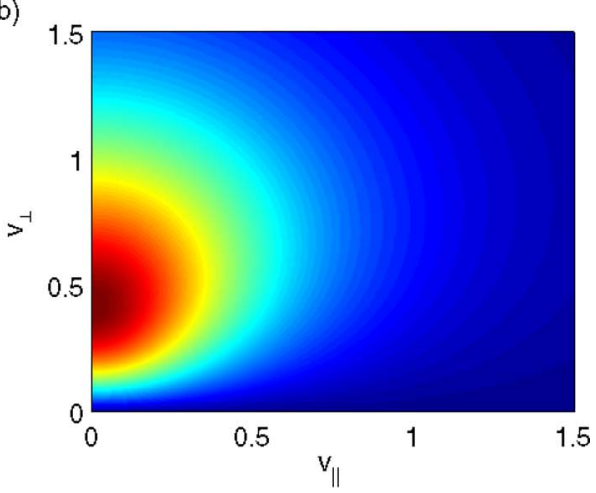

d)

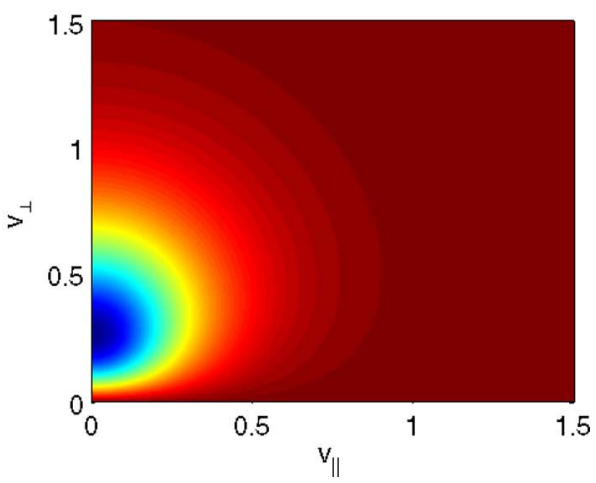

FIG. 5. (Color online) Real (a, b) and imaginary (c, d) part of the ion (a, c) and electron (b, d) distribution functions for the case of $k \rho_{s}=38, L_{n} / R=0.5$, and $\eta=0$, as a function of $v_{\|} / v_{t h}$ and $v_{\perp} / v_{t h}$.

shown in Eq. (B11), predicts that the growth rate has a maximum at $\tau=1$, since the growth rate scales with the temperature ratio $\tau$ as $\tau^{3 / 2}(1+\tau)^{2} /\left(1+\tau^{3}\right)^{2}$.

\section{CONCLUSIONS}

In the present paper, the gyrokinetic model developed in Refs. 14 and 15 is applied to the study of the entropy mode in a $\mathrm{Z}$ pinch. The gyrokinetic equations are solved using the GS2 code and benchmarked with an independent numerical solution in the simplest case. The study considers $\mathrm{Z}$ pinches in the low $\beta$ regime, in the collisionless and collisional cases.

It is shown that the entropy mode is unstable for density gradients lower than the marginal stability for the ideal mode and has a threshold at $L_{n} / R=\pi / 2$ (in the collisionless case and $\eta=0)$. The instability extends over a large span of $k \rho_{s}$ and the peak growth rate, present at $k \rho_{s} \sim 1$, increases strongly with $L_{n} / R$. At high $k$ and low density gradient, high resolution in the velocity grid is required for the correct evaluation of the growth rate. The dependence of the growth rate on the temperature gradient $\eta$ and on collision frequency $\nu$ is studied. Positive temperature gradients lead to a stabilization of the entropy mode, decreasing the peak growth rate and reducing the span of $k \rho_{i}$ where the mode is unstable. The collision frequency $\nu$ reduces the growth rate at high $k$ and the peak growth rate at low gradients. The effect of the temperature ratio is also studied: for $\tau>1$ the peak growth rate of the entropy mode is reduced; for $\tau<1$, the peak growth rate is reduced at low density gradients and it is enhanced at high density gradients.
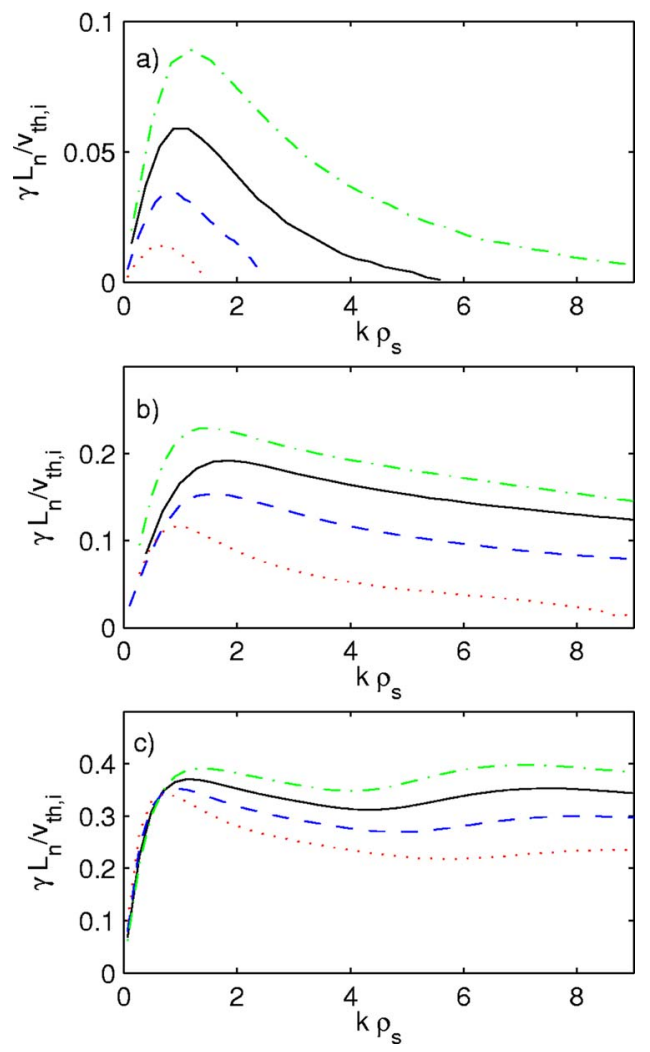

FIG. 6. (Color online) Growth rate of the entropy mode as a function of $k \rho_{s}$ for different values of $L_{n} / R=1$ (a), $L_{n} / R=0.67$ (b), and $L_{n} / R=0.4$ (c), and $\eta=-0.1$ (green dashed-dotted line), $\eta=0$ (black solid line), $\eta=0.1$ (blue dashed line), and $\eta=0.2$ (red dotted line). It is assumed that $m_{i} / m_{e}=1836$, $\tau=1$, and $\nu=0$. 

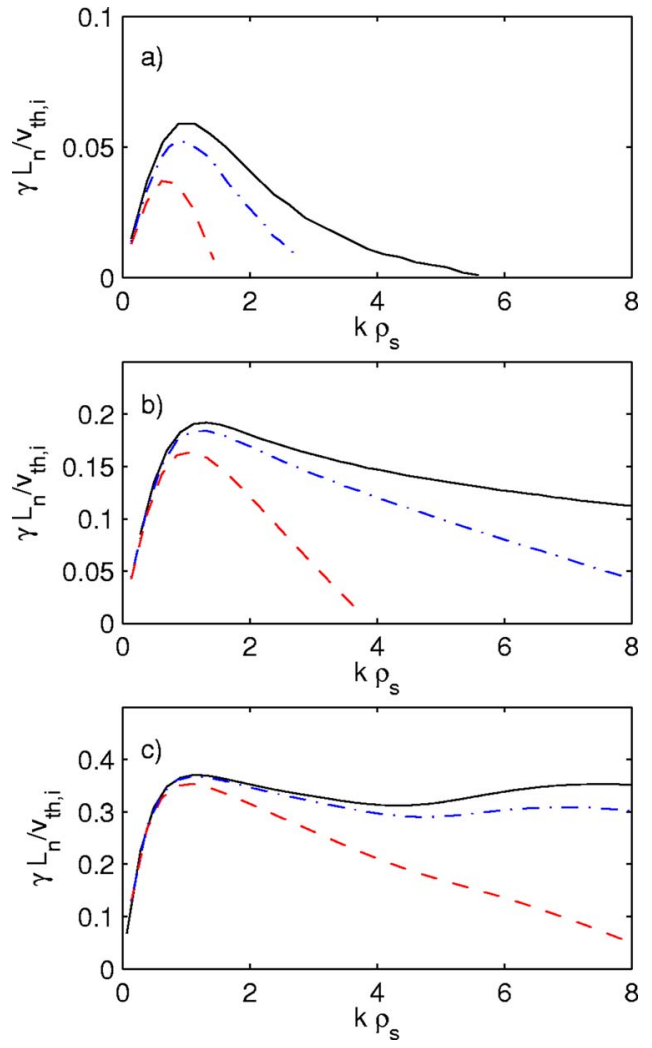

FIG. 7. (Color online) Growth rate of the entropy mode as a function of $k \rho_{s}$ for different values of $L_{n} / R=1$ (a), $L_{n} / R=0.67$ (b), and $L_{n} / R=0.4$ (c), and $\nu=0$ (black solid line), $\nu=0.01 \nu_{t h, i} / R$ (blue dashed-dotted line), $\nu$ $=0.1 \nu_{t h, i} / R$ (red dotted line). It is assumed that $m_{i} / m_{e}=1836, \tau=1$, and $\eta$ $=0$.

The results of our calculations show that the peak growth rate of the entropy mode reaches a value comparable to that of the ideal mode, thus suggesting that the entropy mode may lead to significant particle and heat transport in an ideally stable $\mathrm{Z}$ pinch. In the regime $\tau \gtrsim 1$, a full kinetic study of the entropy mode, as presented here, is needed, since the peak growth rate is reached at $k \rho_{s} \sim 1$ and the mode is unstable over a large span of $k \rho_{s}$. Moreover, fluid estimates are not able to predict the correct stability threshold of the entropy mode: the kinetic calculations show that the entropy mode is unstable at a gradient two times lower than the marginal stability gradient predicted by the fluid theory.

\section{ACKNOWLEDGMENTS}

We thank G. W. Hammett and J. Kesner for useful discussions. The root solver for the energy grid in GS2 used in the present paper has been implemented by T. Tatsuno.

Financial support from the NASA Sun-Earth Connection Theory Program under Grant No. NNG05GJ70G is acknowledged. Support for this project from DOE Center for Multiscale Plasma Dynamics is also acknowledged.

\section{APPENDIX A: GYROFLUID MODEL}

Here we obtain the dispersion relation of the interchange and entropy modes from the gyrofluid model. ${ }^{18-20}$ The gyrofluid model is derived by evaluating the moments of the
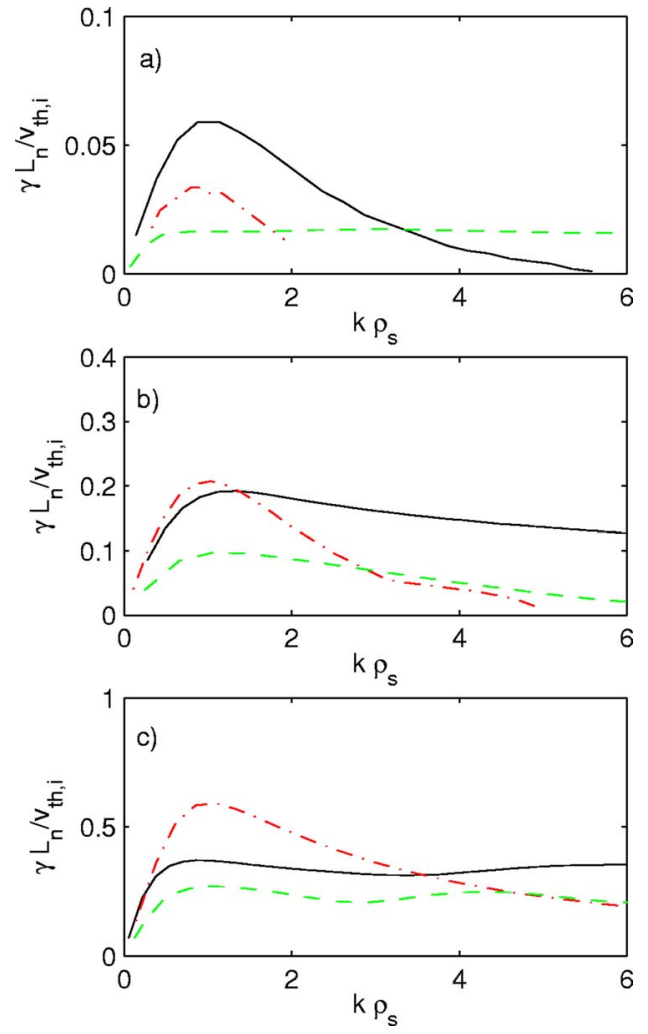

FIG. 8. (Color online) Growth rate of the entropy mode as a function of $k \rho_{s}$ for different values of $L_{n} / R=1$ (a), $L_{n} / R=0.67$ (b), and $L_{n} / R=0.4$ (c), and $T_{0 i} / T_{0 e}=1$ (black solid line), $T_{0 i} / T_{0 e}=0.2$ (red dashed-dotted line), $T_{0 i} / T_{0 e}$ $=5$ (green dashed line). It is assumed that $m_{i} / m_{e}=1836, \nu=0$, and $\eta=0$.

gyrokinetic equations, with the assumptions that the equilibrium distribution functions are Maxwellian and that terms involved in the curvature and $\nabla B$ drifts related to deviations from the Maxwellian distribution functions can be neglected. This approximation is valid for collisional plasmas, where the Maxwellian distribution function is reached through collisions, and for $\omega \gtrsim \omega_{*}$ and $\omega \gg \omega_{d}$ in the collisionless case. We remark that the most general form of the gyrofluid model takes into account deviations of the perturbed distribution functions from Maxwellian distributions. ${ }^{18-20}$ For ions and electrons, the evolution equations for the perturbations of the density, $n_{\alpha}$, and of the parallel and perpendicular pressure, $p_{\| \alpha}$ and $p_{\perp \alpha}$, are

$$
\begin{aligned}
\frac{d n_{i}}{d t}- & \frac{n_{0} e}{T_{0 i}}\left(1+\frac{\eta}{2} \hat{\nabla}_{\perp}^{2}\right) i \omega_{*_{i}} \Psi+\frac{n_{0} e}{T_{0 i}}\left(2+\frac{1}{2} \hat{\nabla}_{\perp}^{2}\right) i \omega_{d i} \Psi \\
& +i \frac{\omega_{d i}}{T_{0 i}}\left(p_{\| i}+p_{\perp i}\right)=0,
\end{aligned}
$$

$$
\begin{aligned}
\frac{d p_{\| i}}{d t} & -n_{0} e\left(1+\eta+\frac{\eta}{2} \hat{\nabla}_{\perp}^{2}\right) i \omega_{*_{i}} \Psi+n_{0} e\left(4+\frac{1}{2} \hat{\nabla}_{\perp}^{2}\right) i \omega_{d i} \Psi \\
& +i \omega_{d i}\left(7 p_{\| i}+p_{\perp i}-4 T_{0 i} n_{i}\right)=-\frac{2}{3} \nu\left(p_{\| i}-p_{\perp i}\right), \quad
\end{aligned}
$$




$$
\begin{aligned}
& \frac{d p_{\perp i}}{d t}-n_{0} e\left[1+\frac{1}{2} \hat{\nabla}_{\perp}^{2}+\eta\left(1+\frac{1}{2} \hat{\nabla}_{\perp}^{2}+\hat{\nabla}_{\perp}^{2}\right)\right] i \omega_{*_{i}} \Psi \\
& +n_{0} e\left(3+\frac{3}{2} \hat{\nabla}_{\perp}^{2}+\hat{\hat{\nabla}}_{\perp}^{2}\right) i \omega_{d i} \Psi+i \omega_{d i}\left(5 p_{\| i}+p_{\perp i}\right. \\
& \left.-3 T_{0 i} n_{i}\right)=\frac{1}{3} \nu\left(p_{\| i}-p_{\perp i}\right), \\
& \frac{d n_{e}}{d t}+\frac{n_{0} e}{T_{0 e}}\left(i \omega_{*_{e}}-2 i \omega_{d e}\right) \phi+i \frac{\omega_{d e}}{T_{0 e}}\left(p_{\| e}+p_{\perp e}\right)=0, \\
& \frac{d p_{\| e}}{d t}+n_{0} e\left[(1+\eta) i \omega_{* e}-4 i \omega_{d e}\right] \phi+i \omega_{d e}\left(7 p_{\| e}+p_{\perp e}\right. \\
& \left.-4 T_{0 e} n_{e}\right)=-\frac{2}{3} \nu\left(p_{\| e}-p_{\perp e}\right), \\
& \frac{d p_{\perp e}}{d t}+n_{0} e\left[(1+\eta) i \omega_{* e}-3 i \omega_{d e}\right] \phi+i \omega_{d e}\left(5 p_{\| e}+p_{\perp e}\right. \\
& \left.-3 T_{0 e} n_{e}\right)=\frac{1}{3} \nu\left(p_{\| e}-p_{\perp e}\right),
\end{aligned}
$$

where we neglect the electron finite Larmor radius effect and where

$$
\hat{\nabla}_{\perp}^{2}=2 b \frac{\partial \Gamma_{0}^{1 / 2}}{\partial b}, \quad \hat{\hat{\nabla}}_{\perp}^{2}=b \frac{\partial^{2}}{\partial b^{2}}\left(b \Gamma_{0}^{1 / 2}\right), \quad \Psi=\Gamma_{0}^{1 / 2} \phi
$$

with $\Gamma_{0}(b)=\exp (-b) I_{0}(b), I_{0}$ is a modified Bessel function, $b=k^{2} \rho_{i}^{2}$, and $\omega_{d \alpha}$ and $\omega_{* \alpha}$ are defined in Eqs. (11) and (12). The system includes the Poisson equation (quasineutrality constraint) that can be written as

$$
n_{e}=\frac{1}{1+b / 2} n_{i}-n_{0} \frac{2 b}{(2+b)^{2}} \frac{T_{\perp i}}{T_{0 i}}-n_{0}\left(1-\Gamma_{0}\right) \frac{e \Psi}{T_{0 i}} .
$$

The gyrofluid model is rigorously first order accurate in $\left(k \rho_{i}\right)^{2}$, since the evaluation of the integrals in velocity space that lead to the $\Gamma_{0}$ function in Eqs. (A1)-(A6) and (A8) and the Padé approximation in Eq. (A8) are accurate to first order in $\left(k \rho_{i}\right)^{2}$. No assumptions are made regarding $k \rho_{s e}{ }^{26}$

The dispersion relation for highly collisional plasmas can be obtained from the set of Eqs. (A1)-(A6) and (A8), in the limit of $\nu \rightarrow \infty$ in Eqs. (A2), (A3), (A5), and (A6). For $\nu \rightarrow \infty$, it follows $p_{\| \alpha}=p_{\perp \alpha}$. Retaining only terms up to the first order in $\left(k \rho_{i}\right)^{2}$, the dispersion relation obtained is a fourth order polynomial,

$$
a_{4} \omega^{4}+a_{3} \omega^{3}+a_{2} \omega^{2}+a_{1} \omega+a_{0}=0,
$$

where

$$
\begin{aligned}
& a_{4}=9\left(k \rho_{s e}\right)^{2}, \\
& a_{3}=\left[-\frac{63}{2} \omega_{d i}-60 \omega_{d e}-9(1+\eta) \omega_{*_{i}}\right]\left(k \rho_{s e}\right)^{2},
\end{aligned}
$$

$$
\begin{aligned}
a_{2}= & \omega_{d e}\left[18(1+\eta)\left(\omega_{*_{e}}-\omega_{*_{i}}\right)+60\left(\omega_{d i}-\omega_{d e}\right)\right]+\left\{3 \omega_{d i}^{2}\right. \\
& \left.+210 \omega_{d i} \omega_{d e}+60 \omega_{d e}^{2}+\omega_{*_{i}}\left[30 \omega_{d i}+60(1+\eta) \omega_{d e}\right]\right\} \\
& \times\left(k \rho_{s e}\right)^{2}, \\
a_{1}= & -60 \omega_{d e}\left[2\left(\omega_{d i}^{2}-\omega_{d e}^{2}\right)-\left(\omega_{d i} \omega_{*_{i}}-\omega_{d e} \omega_{*_{e}}\right)\right] \\
& +10 \omega_{d e} \omega_{d i}\left[2 \omega_{d i}-17 \omega_{d e}-\omega_{*_{i}}(21+\eta)-7 \omega_{*_{e}}\right. \\
& \times(1+\eta)]\left(k \rho_{s e}\right)^{2}, \\
a_{0}= & 40 \omega_{d e}^{2}\left(\omega_{d i}-\omega_{d e}\right)\left[10 \omega_{d i}+\omega_{*_{i}}(3 \eta-7)\right] \\
& +20 \omega_{d e}^{2} \omega_{d i}\left(\omega_{d i}+10 \omega_{*_{i}}\right)\left(k \rho_{s e}\right)^{2} .
\end{aligned}
$$

Assuming that $\omega \sim v_{\text {th }, i} / L$, so that $\omega \gg \omega_{*} \sim \omega_{d}$ for $k \rho_{i}$ $\ll 1$, the collisional (MHD) dispersion relation is derived for the interchange mode, Eq. (2), that is also deduced, e.g., in Ref. 5. If one scales the mode frequency as $\omega \sim \omega_{d} \sim \omega_{*}$, so that $\omega \ll v_{\text {th }, i} / L$, the dispersion relation for the collisional entropy mode is obtained, Eq. (6), which is deduced also, e.g., in Ref. 5.

For collisionless plasmas, $\nu=0$, the dispersion relation obtained is a sixth order polynomial equation in $\omega$,

$$
a_{6} \omega^{6}+a_{5} \omega^{5}+a_{4} \omega^{4}+a_{3} \omega^{3}+a_{2} \omega^{2}+a_{1} \omega+a_{0}=0,
$$

where

$$
\begin{aligned}
a_{6}= & -\left(k \rho_{s e}\right)^{2}, \\
a_{5}= & {\left[9 \omega_{d i}+12 \omega_{d e}+(\eta+1) \omega_{*_{i}}\right]\left(k \rho_{s e}\right)^{2}, } \\
a_{4}= & \omega_{d e}\left[7\left(\omega_{d e}-\omega_{d i}\right)+2(\eta+1)\left(\omega_{*_{i}}-\omega_{*_{e}}\right)\right] \\
& -\left[18 \omega_{d i}^{2}+108 \omega_{d i} \omega_{d e}+41 \omega_{d e}^{2}+12(1+\eta) \omega_{d e} \omega_{*_{i}}\right. \\
& \left.+3 \omega_{d i} \omega_{*_{i}}(3+2 \eta)\right]\left(k \rho_{s e}\right)^{2}, \\
a_{3}= & \omega_{d e}\left[48\left(\omega_{d i}^{2}-\omega_{d e}^{2}\right)-(17+10 \eta)\left(\omega_{d i} \omega_{*_{i}}-\omega_{*_{e}} \omega_{d e}\right)\right] \\
& +\left\{216 \omega_{d i}^{2} \omega_{d e}+369 \omega_{d i} \omega_{d e}^{2}+34 \omega_{d e}^{3}+\omega_{*_{i}}\left[\omega_{d i}^{2}(18\right.\right. \\
& \left.\left.\left.+\frac{3}{2} \eta\right)+\omega_{d i} \omega_{d e}(108+72 \eta)+41 \omega_{d e}^{2}(1+\eta)\right]\right\} \\
& \times\left(k \rho_{s e}\right)^{2}, \\
& \left.+\eta) \omega_{d e} \omega_{*_{e}}\right\}\left(k \rho_{s e}\right)^{2}, \\
& +\omega_{*_{i}}\left[(216+18 \eta) \omega_{d i}+(369+246 \eta) \omega_{d e}\right]+34(1 \\
& +\omega_{d e} \omega_{*_{i}}\left[34 \omega_{d i}^{2}+122 \omega_{d e} \omega_{d i}-122 \omega_{d e}^{2}+38 \eta \omega_{d e}\left(\omega_{d i}\right.\right. \\
& \left.\left.-\omega_{d e}\right)\right]-34 \omega_{d e}^{3} \omega_{* e}-\omega_{d i} \omega_{d e}\left\{306 \omega_{d e}^{2}+738 \omega_{d i} \omega_{d e}\right.
\end{aligned}
$$

$$
\begin{aligned}
a_{1}= & \omega_{d e}^{2}\left[578 \omega_{d i}\left(\omega_{d i}^{2}-\omega_{d e}^{2}\right)+(68 \eta-340) \omega_{*_{i}}\left(\omega_{d i}^{2}-\omega_{d e}^{2}\right)\right] \\
& +\omega_{d e}^{2} \omega_{d i}\left[\omega_{d i} \omega_{*_{i}}\left(738+\frac{123}{2} \eta\right)+612 \omega_{d i} \omega_{d e}\right. \\
& \left.+\omega_{d e} \omega_{*_{i}}(306+204 \eta)\right]\left(k \rho_{s e}\right)^{2},
\end{aligned}
$$




$$
\begin{aligned}
a_{0}= & \left(1156 \omega_{d i}^{2}+340 \eta \omega_{d i} \omega_{*_{i}}-816 \omega_{d i} \omega_{*_{i}}\right) \omega_{d e}^{3}\left(\omega_{d e}-\omega_{d i}\right) \\
& -51(\eta+12) \omega_{d e}^{3} \omega_{d i}^{2} \omega_{*_{i}}\left(k \rho_{s e}\right)^{2} .
\end{aligned}
$$

Again, two analytical limits can be derived from this dispersion relation. If one assumes that $\omega \sim v_{\text {th }, i} / L$ so that $\omega \gg \omega_{*}$ $\sim \omega_{d}$ for $k \rho_{i} \ll 1$, the dispersion relation for the interchange mode is derived. The result can be written as in Eq. (5) and corresponds to that obtained from the CGL model ${ }^{21}$ for $\tau$ $=1{ }^{22}$ If one assumes that the mode frequency scales as $\omega$ $\sim \omega_{d} \sim \omega_{*}$, so that $\omega \ll v_{\text {th }, i} / L$, the dispersion relation for the so-called entropy mode is obtained, Eq. (8). This result is rigorously valid only in the regime $\omega_{*} \gg \omega_{d}$, because terms raising from deviations from the Maxwellian distribution function are neglected in the curvature and $\nabla B$ drifts. ${ }^{18}$

\section{APPENDIX B: EVALUATION OF THE DENSITY GRADIENT AT MARGINAL STABILITY}

In the present Appendix we derive the stability threshold value of the density gradient for the entropy mode from the gyrokinetic model. Since we consider a collisionless plasma, it is $C\left(h_{\alpha}\right)=0$. The dispersion relation of the gyrokinetic model can be deduced from Eqs. (9), (10), and (15), and can be written as

$$
\begin{gathered}
\int \frac{\partial F_{0 e}}{\partial \epsilon} d \mathbf{v}+\int \frac{\partial F_{0 i}}{\partial \epsilon} d \mathbf{v}-\int J_{0}^{2}\left(\frac{k v_{\perp}}{\Omega_{c e}}\right) \frac{\omega-\Omega_{*_{e}}}{\omega-\Omega_{d e}} \frac{\partial F_{0 e}}{\partial \epsilon} d \mathbf{v} \\
-\int J_{0}^{2}\left(\frac{k v_{\perp}}{\Omega_{c i}}\right) \frac{\omega-\Omega_{*_{i}}}{\omega-\Omega_{d i}} \frac{\partial F_{0 i}}{\partial \epsilon} d \mathbf{v}=0 .
\end{gathered}
$$

In order to proceed analytically in the evaluation of the stability threshold, the dispersion relation (B1) is simplified by neglecting electron FLR, so that $J_{0}^{2}\left(k v_{\perp} / \Omega_{c e}\right) \approx 1$, by assuming $k \rho_{i} \ll 1$, so that $J_{0}^{2}\left(k v_{\perp} / \Omega_{c i}\right) \approx 1-k^{2} v_{\perp}^{2} /\left(2 \Omega_{c i}^{2}\right)$, by assuming no temperature gradient, $\eta=0$, and by imposing that $F_{0 \alpha}$ are isotropic Maxwellian distribution functions. With these assumptions, the dispersion relation (B1) becomes

$$
\frac{1}{T_{0 e}}+\frac{1}{T_{0 i}}-I_{e}-I_{i}=0,
$$

where

$$
\begin{aligned}
I_{e}= & \frac{1}{T_{0 e}}\left(\frac{m_{e}}{2 \pi T_{0 e}}\right)^{3 / 2} \int \frac{\omega-\Omega_{*_{e}}}{\omega-\Omega_{d e}} \exp \left(-\frac{m_{e} v^{2}}{2 T_{0 e}}\right) d \mathbf{v}, \\
I_{i}= & \frac{1}{T_{0 i}}\left(\frac{m_{i}}{2 \pi T_{0 i}}\right)^{3 / 2} \int\left(1-\frac{k^{2} v_{\perp}^{2}}{2 \Omega_{c i}^{2}}\right) \frac{\omega-\Omega_{*_{i}}}{\omega-\Omega_{d i}} \\
& \times \exp \left(-\frac{m_{i} v^{2}}{2 T_{0 i}}\right) d \mathbf{v} .
\end{aligned}
$$

We consider first $I_{e}$. Since $\eta=0$, it follows $\Omega_{*_{e}} / \omega_{*_{e}}=1$. Dividing and multiplying $I_{e}$ by $\omega_{*}$, one obtains

$$
\begin{aligned}
I_{e}= & \frac{2 \pi R\left(\omega / \omega_{*_{e}}-1\right)}{m_{e} L_{n}}\left(\frac{m_{e}}{2 \pi T_{0 e}}\right)^{3 / 2} \int_{0}^{\infty} d v_{\perp} v_{\perp} \\
& \times \exp \left(-\frac{m_{e} v_{\perp}^{2}}{2 T_{0 e}}\right) \int_{-\infty}^{\infty} d v_{\|} \frac{\exp \left(-m_{e} v_{\|}^{2} / 2 T_{0 e}\right)}{\omega v_{\mathrm{th}, e}^{2} / \omega_{d e}-v_{\perp}^{2} / 2-v_{\|}^{2}},
\end{aligned}
$$

where cylindrical velocity coordinates and the definition of $\Omega_{d e}$ in Eq. (12) are used. The analytical integration in $v_{\|}$ yields

$$
\begin{aligned}
\int_{-\infty}^{\infty} & d v_{\|} \frac{\exp \left(-m_{e} v_{\|}^{2} / 2 T_{0 e}\right)}{\omega v_{\mathrm{th}, e}^{2} / \omega_{d e}-v_{\perp}^{2} / 2-v_{\|}^{2}} \\
= & \exp \left(m_{e} \frac{v_{\perp}^{2} / 2-\omega v_{\mathrm{th}, e}^{2} / \omega_{d e}}{2 T_{0 e}}\right) \\
& \times \frac{\pi}{\sqrt{\omega v_{\mathrm{th}, e}^{2} / \omega_{d e}-v_{\perp}^{2} / 2}} i\left\{\operatorname{sgn}\left[\operatorname{Im}\left(-\omega / \omega_{*}\right)\right]\right. \\
& \left.-\operatorname{erf}\left(i \sqrt{m_{e} \frac{\omega v_{\mathrm{th}, e}^{2} / \omega_{d e}-v_{\perp}^{2} / 2}{2 T_{0 e}}}\right)\right\}
\end{aligned}
$$

defining $\operatorname{sgn}(x)=1$ if $x>0$, and $\operatorname{sgn}(x)=-1$ if $x<0$. The result of the $v_{\|}$integration, Eq. (B6), is introduced in Eq. (B5), obtaining

$$
\begin{aligned}
I_{e}= & \frac{2 \pi\left(\omega / \omega_{*_{e}}-1\right) R}{m_{e} L_{n}}\left(\frac{m_{e}}{2 \pi T_{0 e}}\right)^{3 / 2} \\
& \times \exp \left(-\frac{\omega}{2 \omega_{d e}}\right) \int_{0}^{\infty} d v_{\perp} v_{\perp} \exp \left(-\frac{m_{e} v_{\perp}^{2}}{4 T_{0 e}}\right) \\
& \times \frac{i \pi}{\sqrt{\omega v_{\mathrm{th}, e}^{2} / \omega_{d e}-v_{\perp}^{2} / 2}}\left\{\operatorname{sgn}\left[\operatorname{Im}\left(-\omega / \omega_{*}\right)\right]\right. \\
& -\operatorname{erf}\left(i \sqrt{\left.\left.m_{e} \frac{\omega v_{\mathrm{th}, e}^{2} / \omega_{d e}-v_{\perp}^{2} / 2}{2 T_{0 e}}\right)\right\}}\right. \\
= & \frac{\pi}{2} \frac{R}{L_{n}} \frac{\left(1-\omega / \omega_{* e}\right)}{T_{0 e}} \exp \left(-\frac{\omega}{\omega_{d e}}\right)\left[\operatorname{erfc}\left(\sqrt{-\frac{\omega}{2 \omega_{d e}}}\right)\right]^{2} .
\end{aligned}
$$

For the ions, the integral to be evaluated can be written as

$$
\begin{aligned}
I_{i}= & \frac{2 \pi\left(\omega / \omega_{*_{e}}+\tau\right) R}{\tau m_{i} L_{n}}\left(\frac{m_{i}}{2 \pi T_{0 i}}\right)^{3 / 2} \int_{0}^{\infty} d v_{\perp} v_{\perp}\left(1-\frac{k^{2} v_{\perp}^{2}}{2 \Omega_{c i}^{2}}\right) \\
& \times \exp \left(-\frac{m_{i} v_{\perp}^{2}}{2 T_{0 i}}\right) \int_{-\infty}^{\infty} d v_{\|} \frac{\exp \left(-m_{i} v_{\|}^{2} / 2 T_{0 i}\right)}{\omega v_{\mathrm{th}, i}^{2} /\left(\omega_{d e} \tau\right)+v_{\perp}^{2} / 2+v_{\|}^{2}} .
\end{aligned}
$$

The integral in $v_{\|}$leads to 


$$
\begin{aligned}
\int_{-\infty}^{\infty} & d v_{\|} \frac{\exp \left(-m_{i} v_{\|}^{2} / 2 T_{0 i}\right)}{\omega v_{\mathrm{th}, i}^{2} /\left(\omega_{d e} \tau\right)+v_{\perp}^{2} / 2+v_{\|}^{2}} \\
= & \exp \left(m_{i} \frac{\omega v_{\mathrm{th}, i}^{2} /\left(\omega_{d e} \tau\right)+v_{\perp}^{2} / 2}{2 T_{0 i}}\right) \frac{\pi}{\sqrt{\omega v_{\mathrm{th}, i}^{2} /\left(\omega_{d e} \tau\right)+v_{\perp}^{2} / 2}} \\
& \times\left[\operatorname{erfc}\left(\sqrt{m_{i} \frac{\omega v_{\mathrm{th}, i}^{2} /\left(\omega_{d e} \tau\right)+v_{\perp}^{2} / 2}{2 T_{0 i}}}\right)\right]
\end{aligned}
$$

and, using Eq. (B9) to evaluate the $v_{\perp}$ integral in Eq. (B8), the following expression for $I_{i}$ is obtained:

$$
\begin{aligned}
I_{i}= & \frac{1}{\sqrt{2 \pi} T_{0 i}}\left(\frac{\omega}{\omega_{* e} \tau}+1\right) \frac{R}{L_{n}} \\
& \times\left\{\exp \left(\frac{\omega}{\tau \omega_{d e}}\right) \sqrt{\frac{\pi^{3}}{2}}\left[\operatorname{erfc}\left(\sqrt{\frac{\omega}{2 \tau \omega_{d e}}}\right)\right]^{2}\right. \\
& -\frac{k^{2} \rho_{i}^{2}}{2}\left[-2 \sqrt{2 \pi}+4 \pi \exp \left(\frac{\omega}{2 \tau \omega_{d e}}\right)\right. \\
& \times \sqrt{\frac{\omega}{\tau \omega_{d e}}} \operatorname{erfc}\left(\sqrt{\frac{\omega}{2 \tau \omega_{d e}}}\right)-\sqrt{2 \pi^{3}} \\
& \left.\left.\times \exp \left(\frac{\omega}{\tau \omega_{d e}}\right)\left(\frac{\omega}{\tau \omega_{d e}}-1\right)\left(\operatorname{erfc}\left(\sqrt{\frac{\omega}{2 \tau \omega_{d e}}}\right)\right)^{2}\right]\right\} .
\end{aligned}
$$

Introducing Eqs. (B7) and (B10) into the dispersion relation, Eq. (B2), and solving for $\omega$, it is possible to evaluate the growth rate and real frequency of the entropy mode. Since we are interested in evaluating the marginal stability of the mode, it is possible to assume $\omega \ll \omega_{*}$. Taylor expanding the dispersion relation in powers of $\omega$, we obtain

$$
\begin{aligned}
\omega= & k \rho_{i} \frac{v_{\mathrm{th}, i}}{L_{n}} \frac{(1+\tau)\left[\pi R /\left(2 L_{n}\right)-1\right]-k^{2} \rho_{i}^{2} R(\pi / 2-1) / L_{n}}{2 \pi\left(\tau^{3}+1\right)^{2}\left(R / L_{n}\right)^{3}} \\
& \cdot\left\{\left[(1+\tau)\left(\frac{\pi R}{2 L_{n}}-1\right)-k^{2} \rho_{i}^{2} \frac{R}{L_{n}}(\pi / 2-1)\right]\left(\tau^{2}-1\right)\right. \\
& \left.+2 \tau^{3 / 2}\left|(1+\tau)\left(\frac{\pi R}{2 L_{n}}-1\right)-k^{2} \rho_{i}^{2} \frac{R}{L_{n}}(\pi / 2-1)\right| i\right\}
\end{aligned}
$$

$$
\frac{L_{n}}{R}=\frac{\pi(1+\tau)-k^{2} \rho_{i}^{2}(\pi-2)}{2(1+\tau)} .
$$

From Eq. (B12), it can be argued that the entropy mode is stable for $L_{n} / R<\pi / 2$ in collisionless plasmas, and that ion FLR effects are stabilizing. The stability threshold is independent of the temperature ratio, but the growth rates depend on $\tau$, as pointed out in Eq. (B11). Moreover, for $\tau=1$, the mode is purely growing.

${ }^{1}$ J. P. Freidberg, Ideal Magnetohydrodynamics (Plenum, New York, 1987).

${ }^{2}$ A. N. Simakov, R. J. Hastie, and P. J. Catto, Phys. Plasmas 9, 201 (2002).

${ }^{3}$ A. B. Hassam and Y. C. Lee, Phys. Fluids 27, 438 (1984).

${ }^{4}$ H. O. Akerstedt, J. Plasma Phys. 44, 137 (1990).

${ }^{5}$ J. Kesner, Phys. Plasmas 7, 3837 (2000).

${ }^{6}$ J. Kesner and R. J. Hastie, Phys. Plasmas 9, 395 (2002).

${ }^{7}$ A. N. Simakov, P. J. Catto, and R. J. Hastie, Phys. Plasmas 8, 4414 (2001).

${ }^{8}$ M. N. Rosembluth and C. L. Longmire, Ann. Phys. (N.Y.) 1, 120 (1957).

${ }^{9}$ I. B. Bernstein, E. Frieman, M. Kruskal, and R. Kulsrud, Proc. R. Soc. London, Ser. A 244, 17 (1998).

${ }^{10}$ B. B. Kadomtsev, Sov. Phys. JETP 10, 780 (1960).

${ }^{11}$ A. A. Ware, Nucl. Fusion Suppl. 3, 869 (1962).

${ }^{12}$ L. V. Mikhailovskaya, Sov. Phys. Tech. Phys. 12, 1451 (1968).

${ }^{13}$ A. B. Mikhailovskii and V. S. Tsypin, Sov. Phys. JETP 32, 287 (1971).

${ }^{14}$ T. M. Antonsen and B. Lane, Phys. Fluids 23, 1205 (1980).

${ }^{15}$ E. A. Frieman and L. Chen, Phys. Fluids 25, 502 (1982).

${ }^{16}$ M. Kotschenreuther, G. Rewoldt, and W. M. Tang, Comput. Phys. Commun. 88, 128 (1995).

${ }^{17}$ W. Dorland, F. Jenko, M. Kotschenreuther, and B. N. Rogers, Phys. Rev. Lett. 85, 5579 (2000).

${ }^{18}$ M. A. Beer, S. C. Cowley, and G. W. Hammett, Phys. Plasmas 2, 2687 (1995).

${ }^{19}$ W. Dorland and G. W. Hammett, Phys. Fluids B 5, 812 (1993).

${ }^{20}$ P. B. Snyder, Ph.D. thesis, Princeton University, Princeton (1999).

${ }^{21}$ C. F. Chew, M. L. Goldberg, and F. E. Low, Proc. R. Soc. London, Ser. A 236, 112 (1956).

${ }^{22}$ M. Coppins, Phys. Fluids B 1, 591 (1989).

${ }^{23}$ T. F. Coleman and Y. Li, Math. Program. 67, 189 (1994).

${ }^{24}$ T. Tatsuno (private communication, 2006).

${ }^{25}$ W. H. Press, B. P. Flannery, S. A. Teukolsky, and W. T. Vetterling, Numerical Recipes (Cambridge University Press, Cambridge, 1988).

${ }^{26}$ B. N. Rogers and W. Dorland, Phys. Plasmas 12, 062511 (2005).

and thus a critical gradient of 